\title{
Computational intelligence for multimedia and industrial applications
}

\author{
Gwanggil Jeon $^{1} \cdot$ Ernesto Damiani $^{2} \cdot$ Marco Anisetti $^{3}$
}

Published online: 7 September 2017

(C) Springer Science+Business Media, LLC 2017

Computational intelligence approaches such as neural network, particle swarm optimization, evolutionary algorithm, fuzzy set, and rough sets are adopted in many multimedia and industrial applications, like visual based quality control, image enhancement in consumer electronics, video based recognition of identity or behaviors, audio based speech recognition for enhanced human like interaction with machines etc. Despite this growing diffusion, there are still many possible areas where computational intelligence application is partial or could be extended and improved, due to the actual limitations in terms of computational power or strict requirements in terms of assurance of the results.

This special issue aims to investigate the impact of the adoption of advanced and innovative Computational Intelligence techniques in multimedia and industrial applications. Authors are expected to discuss some representative applications to present inspiring models to demonstrate how computational intelligence could be implemented to solve multimedia computing and industrial issues and how these issues could be analyzed, processed, and illustrated by computational intelligence. This special issue brings together researchers on different disciplines from academia and industry with a common objective: go beyond the frontiers of today multimedia applications of computational intelligence techniques. The articles contained in the present issue include both reviews and basic scientific studies focused on recent advances in computational intelligence for multimedia and industrial applications. This issue comprises the description of multimedia and industrial applications which uses computational intelligence techniques.

Gwanggil Jeon

gjeon@inu.ac.kr

Ernesto Damiani

ernesto.damiani@kustar.ac.ae

Marco Anisetti

marco.anisetti@unimi.it

1 Incheon National University, Incheon, South Korea

2 EBTIC/Khalifa University, Abu Dhabi, UAE

3 Università degli studi di Milano, Milan, Italy 
Active noise control approach reduces an unwanted noise by generating an anti-noise to make its magnitude equal and phase opposite each other. The contribution by Ryu et al. "Active Sound Profiling of Narrowband Signals for Improving Sound Quality in an Enclosed Space" (https://doi.org/10.1007/s11042-017-4648-3) proposes an active sound profiling (ASP) system which is based on the command-FxLMS algorithm. The control system consists of an error microphone at a control point, a midrange loudspeaker and a subwoofer in the enclosed space with a digital signal processing board. The proposed system was implemented in an enclosed space for improving sound quality. This result showed that the ASP algorithm in the control experiment improved sound quality greatly.

In HEVC, deblocking filtering (DF) is responsible for about $20 \%$ of the time consumed to perform video compression. In a typical parallel DF scheme, a set of horizontal and vertical edges are processed using deblocking filters. The contribution by Fouad and Dansereau "An Optimized Parallel Order Scheme of the Deblocking Filtering Process for Enhancing the Performance of the HEVC Standard Using GPUs" (https://doi.org/10.1007/s11042-0174876-6) proposed an optimized parallel DF scheme for HEVC using graphical processing units. The proposed scheme outperforms conventional methods in terms of reducing the decoding time of all frames of video sequences. In addition, the proposed method does not change the rate-distortion between the decoded video sequences and their original sequences.

In the course of the filming of infrared (IR) video, intrinsic equipment instability incurs movement that in turn causes image blurring. For image clarity and viewing comfortability, it is required that such movement be countered. However, frame-by-frame comparison for longdistance filming often is difficult due to lack of information. The contribution by Kang and Park "Motion-estimation-based Stabilization of Infrared Video" (https://doi.org/10.1007 /s11042-017-4647-4) suggest the most appropriate blocks with the most information for motion estimation. In addition, to stabilize videos, the proposed method discerns equipment movement and movement in the video itself. Based on this method, the proposed method afforded a $10 \%$ increase in accuracy.

Vertical handover gain significant importance due to the enhancements in mobility models by the Fourth Generation (4G) technologies. However, these enhancements are limited to specific scenarios and hence do not provide support for generic mobility. To address this issue, the contribution by Khan et al. "Fuzzy based Multi-criteria Vertical Handover Decision Modeling in Heterogeneous Wireless Networks" (https://oi.org/10.1007/s11042-016-4330-1) proposed a generic vertical handover management scheme for heterogeneous wireless networks is proposed in this article. The proposed scheme is tested in a mobility scenario with different speeds of a mobile node ranging from very low to very high. The proposed scheme outperforms these schemes in terms of energy consumption, handover delay and time, packet loss, good put, etc.

Basmati, Pakistan, is one of the most harvested and most profitable varieties of rice because of its unique fragrance. Rice varieties are difficult to differentiate accurately by visual inspection. Therefore, dishonest dealers could easily mislabel or adulterate basmati rice with less valuable assortments that look similar. The contribution by Ali et al. "Detecting Fraudulent Labeling of Rice Samples Using Computer Vision and Fuzzy Knowledge” (https://doi. org/10.1007/s11042-017-4472-9) proposed a novel and inexpensive method to detect fraudulent labeling by using computer vision and a fuzzy classification database. The proposed method achieves a precision of more than $90 \%$ in identifying fraudulent labels of rice.

Change detection is a fundamental task in the interpretation and understanding of remote sensing images. The aim is to partition the difference images acquired from multi-temporal satellite images into changed and unchanged regions. The contribution by Lei et al. "Region- 
driven Distance Regularized Level Set Evolution for Change Detection in Remote Sensing Images" (https://doi.org/10.1007/s11042-017-4650-9) introduces a distance regularization method using energy function. The energy function can maintain a desired shape of the level set function and keep a signed distance profile near the zero level set. Simulation was tested on remote sensing images, where advantages of the proposed method was demonstrated.

In the contribution by Gianini and Rizzi. "A Fuzzy Set Approach to Retinex Spray Sampling" (https://doi.org/10.1007/s11042-017-4877-5), authors argue that several elements, inherent to the above observation, can benefit from a fuzzy formalization. They show that the adoption of the fuzzy formalism allows to better encode the mutual influence of pixels. In addition, the authors demonstrate its use by the construction of a fuzzy version of the pointsampling algorithm Random Spray Retinex (RSR). By using RSR as a guide, the authors build a more efficient algorithm.

Visual sensor networks require low power compression techniques of large amount of video data in each camera node due to the energy-constrained and bandwidth-limited environments. The contribution by Lee "Energy Efficient Processing of Motion Estimation for Embedded Multimedia Systems" (https://doi.org/10.1007/s11042-017-4645-6) proposes an energyefficient architecture for variable block size motion estimation. The partial reconfiguration of FPGA is exploited to support run-time reconfiguration of the proposed modular hardware architecture for motion estimation. According to the required search range, hardware reconfiguration is performed adaptively to reduce the hardware resources and power consumption.

Social media has drastically entered into a new concept by empowering people to publish their data along with their locations in order to provide benefits to the community and the country overall. There are millions of geosocial network users who generate terabytes of heterogeneous data with a variety of information every day and at high speed; such information is called Big Data. The contribution by Rathore et al. "Advanced Computing Model for Geosocial Media Using Big Data Analytics” (https://doi.org/10.1007/s11042-017-4644-7) proposes an efficient system for exploring geosocial networks while harvesting data in order to make real-time decisions while detecting various events. The authors prove that the proposed system has higher throughput and is capable of analyzing a huge amount of geosocial network data at a real time while detecting any event.

High-quality thermal infrared (IR) images are always preferred in numerous real-world applications. However, acquired IR images, which have low contrast and signal-to-noise ratio (SNR) among other characteristics, have inferior quality because of various factors. The contribution by $\mathrm{Wu}$ et al. "A Novel Scheme for Infrared Image Enhancement by using Weighted Least Squares Filter and Fuzzy Plateau Histogram Equalization" (https://doi. org/10.1007/s11042-017-4643-8) proposes a novel method to solve conventional issues. The authors propose a fuzzy plateau histogram equalization for the LF component to improve global contrast. In addition, to suppress the noise in the primitive result, non-local means filter is applied to derive the final result.

Edges can be described as local intensity changes in an image, and edges generally occur on the border between two distinctive areas. In the field of digital image processing, edge detection approaches have been proven to be able to solve various pattern recognition issues. The contribution by Choi et al. "Rule-Based Soft Computing for Edge Detection" (https://doi.org/10.1007/s11042-016-4329-7) proposes a rule based edge detection method. The purpose of this method is to provide more reliable edge detection results that are effective in most images. The authors used three benchmarks approaches to compare the subjective performance quality. 
To date, continuity editing has been the film director's responsibility. But if continuity editing is used as a means to achieve natural narrative immersion of an audience, technologies must be adopted to analyze viewers' psychological distances based on the changes in their autonomic nervous system, which can then be used in editing. The contribution by Kim and Lee "Audience Real-time Bio-signal-processing-based Computational Intelligence Model for Narrative Scene Editing” (https://doi.org/10.1007/s11042016-4144-1) presents an algorithm for continuity editing that determines the size of a shot (field of view) by evaluating the psychical distance between the characters and viewers via the measurement of electrodermal activity.

Deinterlacing converts interlaced signals into progressive signals by upsampling the nontransmitted lines. Video deinterlacing employs an image enhancement technique that is important in various image processing areas, one of which is format conversion. The contribution by Chyung et al. "Weighted Aggregation and Fuzzy-Concept-Guided Signal Resemblance and Expansion for Video Format Conversion" (https://doi.org/10.1007/s11042-017-4641-x) propose an efficient deinterlacing method for HDTV that preserves image structures, edges, and details. The proposed method is a weighted filtering approach that generates a half-pixel 9by-9 edge-based line average window. The authors also propose pixel-resemblance- and pixelexpansion-based fuzzy weights, which are assigned using a triangular membership function.

Synthetic aperture radar (SAR) techniques have played a crucial role in both military and civilian fields nowadays. A single polarization SAR imaging system cannot provide a large amount of target and surface information which contained in polarization scattering characteristics. The contribution by Bai et al. "PolSAR image compression based on online sparse KSVD dictionary learning" (https://doi.org/10.1007/s11042-017-4640-y) presents a novel polarimetric synthetic aperture radar (PolSAR) image compression scheme. The discrete cosine transform (DCT) is adopted to remove redundancies between polarimetric channels, simple but quite efficient in improving compressibility. In addition, the sparse K-singular value decomposition (K-SVD) dictionary learning algoTrithm is utilized to remove redundancies within each channel image.

Super-resolution (SR) methods are effective for generating a high-resolution image from a single low-resolution image. However, there are few issues that should be solved for SR methods. The contribution by Yang et al. "Multi-sensor Image Super-resolution with Fuzzy Cluster by Using Multi-scale and Multi-view Sparse Representation for Infrared Image" (https://doi.org/10.1007/s11042-017-4639-4) proposes a novel SR method for infrared images. The proposed method has four steps. In the proposed method, clustering information for low-resolution patches is learnt by using fuzzy clustering theory.

The short-term prediction of EDH time series plays an exceedingly important role in several fields such as communications and navigation. The contribution by Yang et al. "The short-term forecasting of evaporation duct height (EDH) based on ARIMA model" (https://doi. org/10.1007/s11042-016-4143-2) presents an application of autoregressive integrated moving average (ARIMA) for short-term forecasting of EDH time series. In order to obtain the EDH, a body of sensors such as air temperature, relative humidity and pressure sensors are installed at different height based on the tower platform. EDH is calculated according to Debye theory and a log-squares curve fit.

Speech enhancement is crucial in various speech communication systems including robust speech recognition, mobile communication, and hearing aids in virtual realities due to ambient acoustic noise. However, the performance of a speech enhancement system deteriorates in non-stationary and low signal-to-noise ratio (SNR) noise environments. The contribution by 
Lee and Chang "Spectral Difference for Statistical Model-Based Speech Enhancement in Speech Recognition" (https://doi.org/10.1007/s11042-016-4122-7) proposes a statistical model-based speech enhancement technique using the spectral difference scheme for the speech recognition in virtual reality. In the analyzing step, two principal parameters are uniquely determined as optimal operating points according to the spectral difference under various noise conditions. In the on-line speech enhancement step, different parameters are chosen on a frame-by-frame basis under the metric table of the spectral difference.

Liver segmentation in medical images is important for surgical operations planning. It needs proper identification of the organ and it helps to overcome the overlapping of the intensity values with other organs. The contribution by Mostafa et al. "Liver segmentation in MRI images based on whale optimization algorithm" (https://doi.org/10.1007/s11042-017-4638-5) proposes an approach for liver segmentation in MRI images based on Whale optimization algorithm. It is used to extract the different clusters in the abdominal image to support the segmentation process. The proposed approach is tested using a set of 70 MRI images, annotated and approved by radiology specialists.

We hope that this special issue would shed light on major developments in the area of computational intelligence for multimedia and industrial applications and attract attention by the scientific community to pursue further investigations leading to the rapid implementation of these technologies.

This special issue was proposed in the framework of International Cooperation Program managed by "Ministero degli Affari Esteri e della Cooperazione Internazionale" of Italy under Grant PGR00217 and by NRF Korea (2016K1A3A1A25003543).

Acknowledgments We would like to express our appreciation to all the authors for their informative contributions and the reviewers for their support and constructive critiques in making this special issue possible. 